Supporting information

Higher-temperature dielectric molecular motor induced by unusual chair-to-rotator motion

Zhi-Xu Zhang, Tie Zhang, Ping-Ping Shi, Wan-Ying Zhang, Qiong Ye* and Da-Wei Fu*

(Ordered Matter Science Research Center, Jiangsu Key Laboratory for Science and Applications of Molecular Ferroelectrics, Southeast University, Nanjing, 211189, P. R. China)

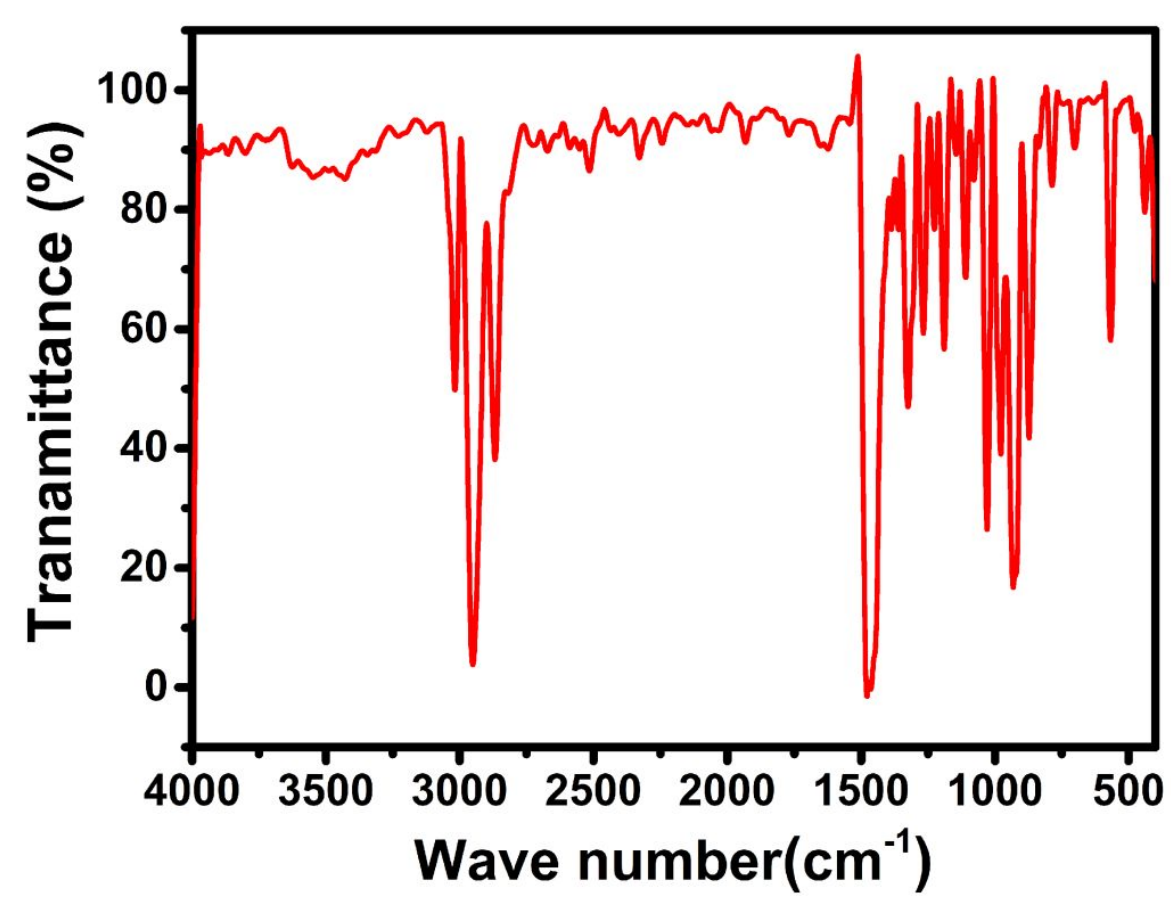

Figure S1. Infrared spectrum of compound 1. 


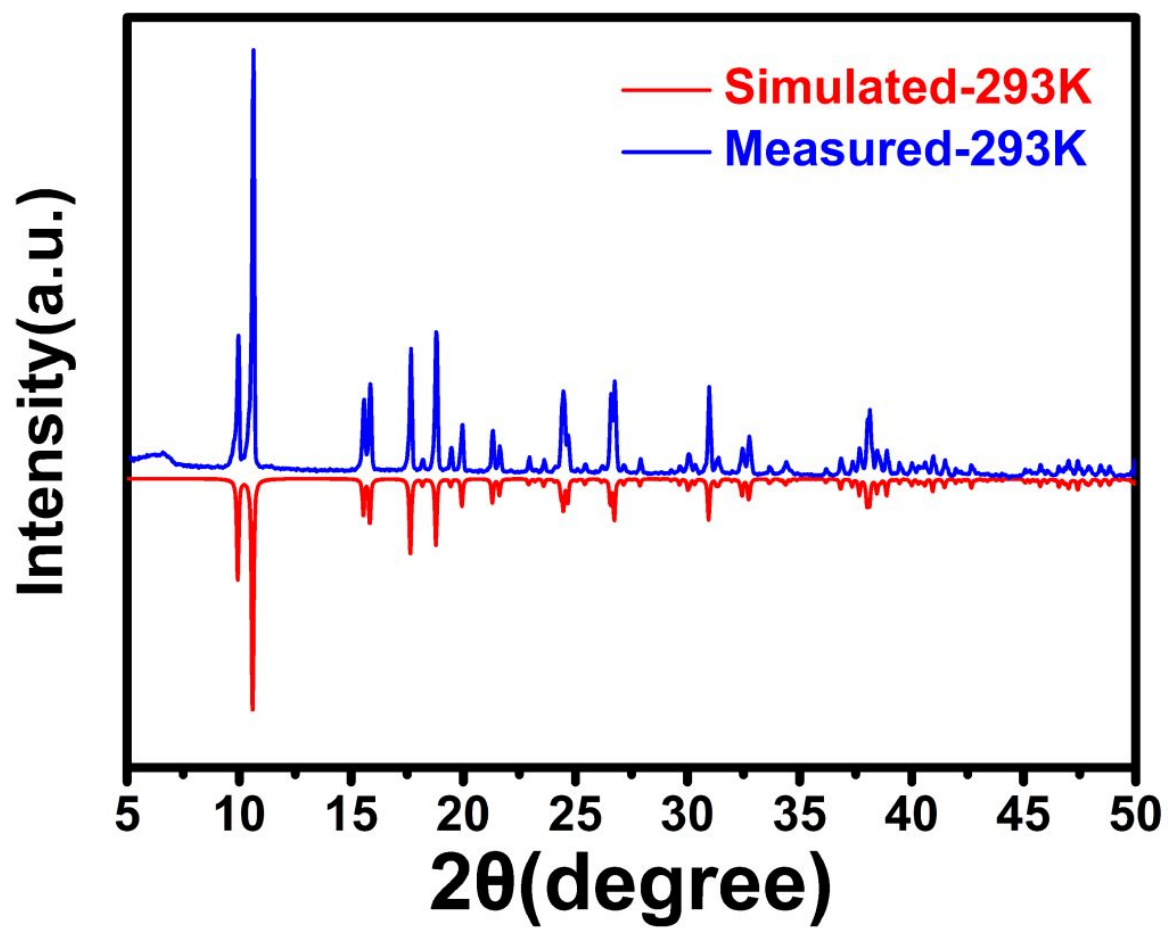

Figure S2. Measured and simulated powder X-ray diffraction patterns of compound 1 at $293 \mathrm{~K}$.

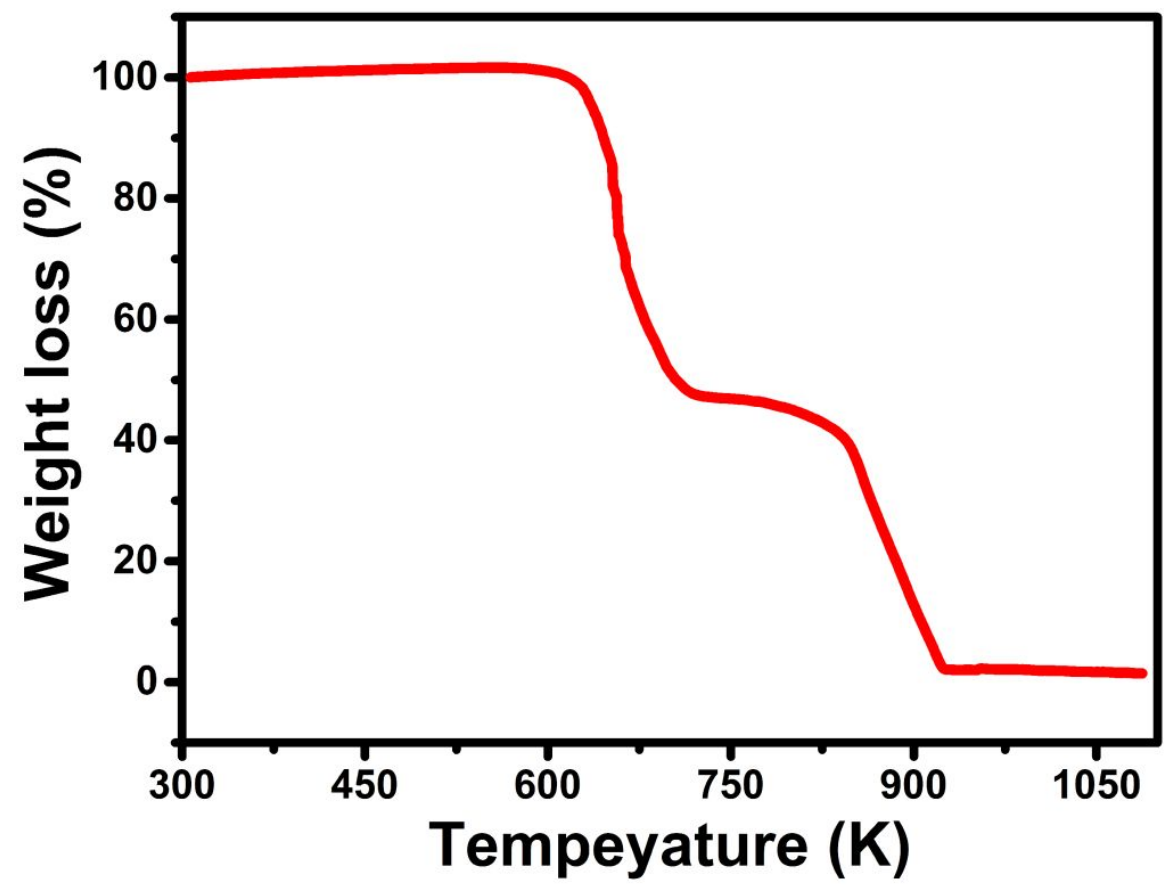

Figure S3. TGA curve of $\mathbf{1}$. 


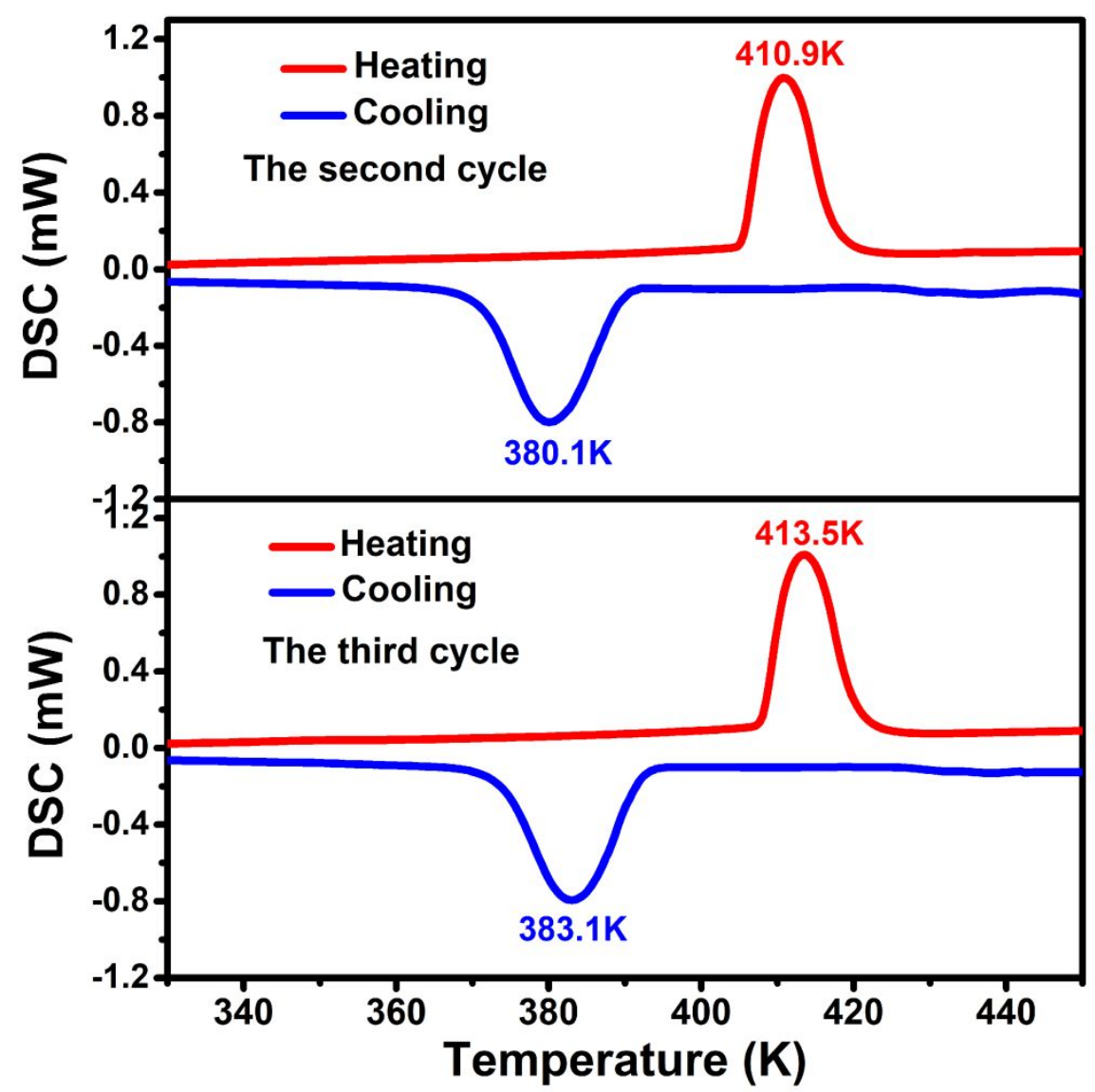

Figure S4. DSC curves of 1 in a cooling-heating run at the second cycle and the third cycle respectively.
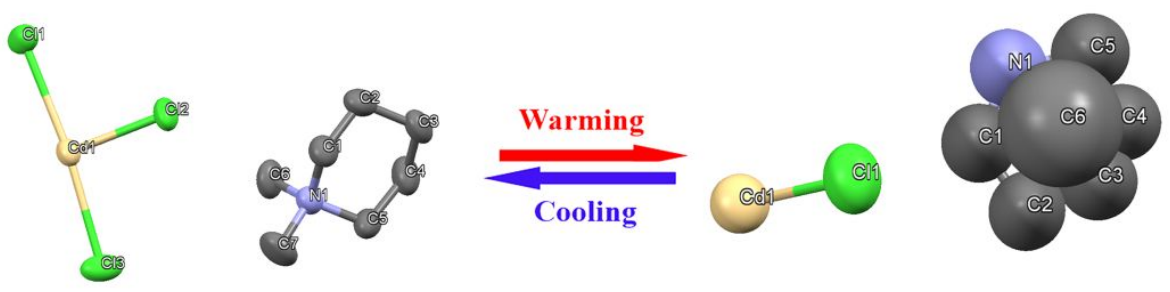

Figure S5. The asymmetric units of $\mathbf{1}$ at LTP and HTP. 

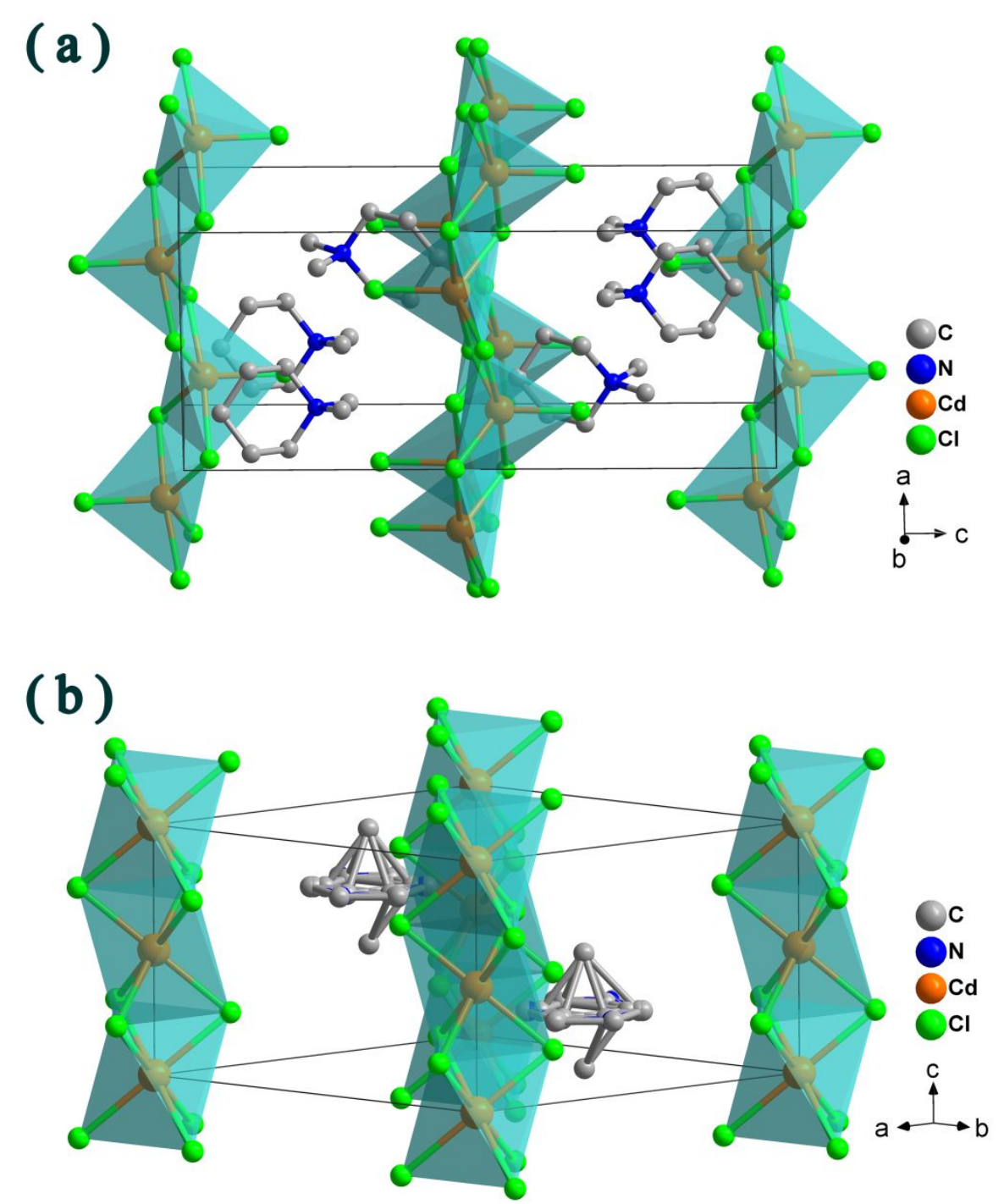

Figure S6. Packing views of 1 at (a) $293 \mathrm{~K}$ and (b) $418 \mathrm{~K}$, where NNDP cations are located in the cavities between the one-dimensional chains, displaying different states of motion. Inorganic anions exhibit different hexahedral and octahedral configurations at $293 \mathrm{~K}$ and $418 \mathrm{~K}$, respectively. All the hydrogen atoms are omitted for clarity. 


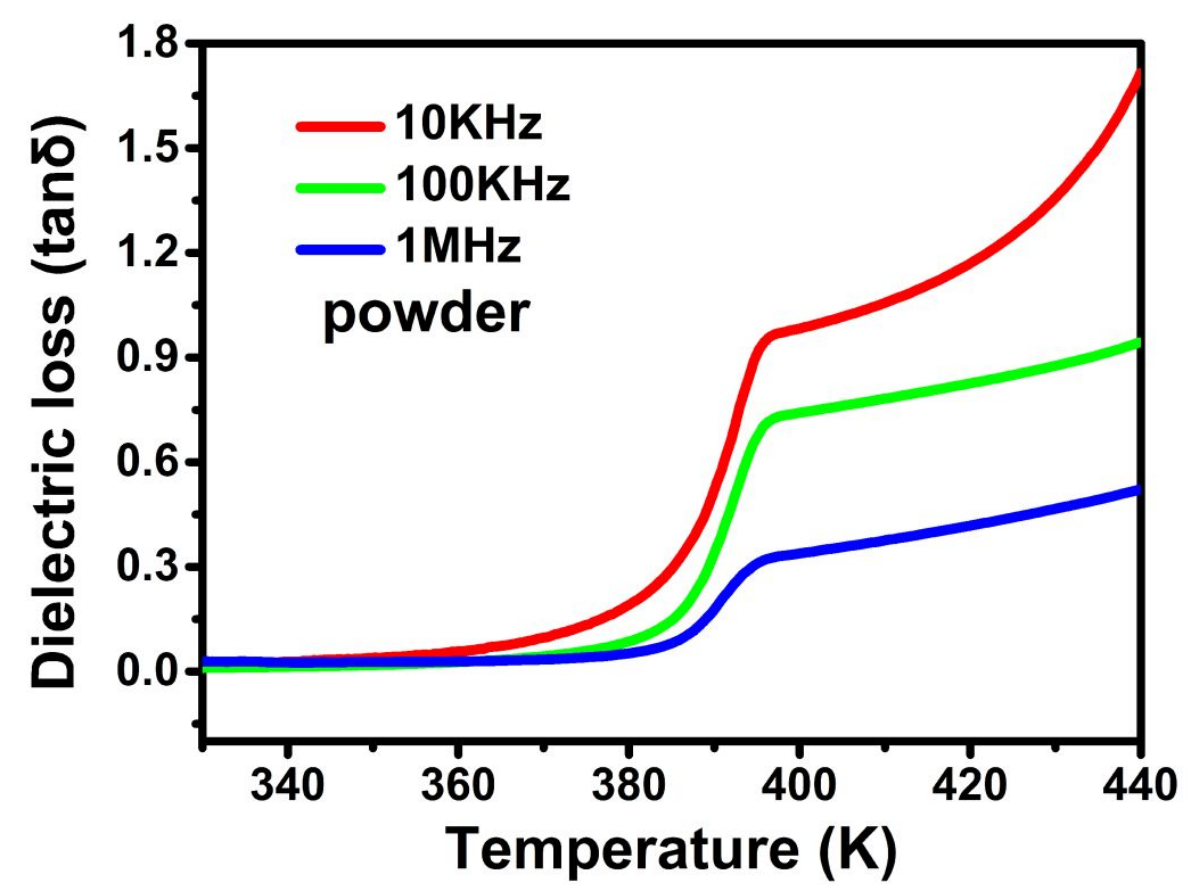

Figure S7. Dielectric loss ( $\tan \delta$ ) of the dielectric constant of compound 1 measured at selected frequencies in cooling mode.

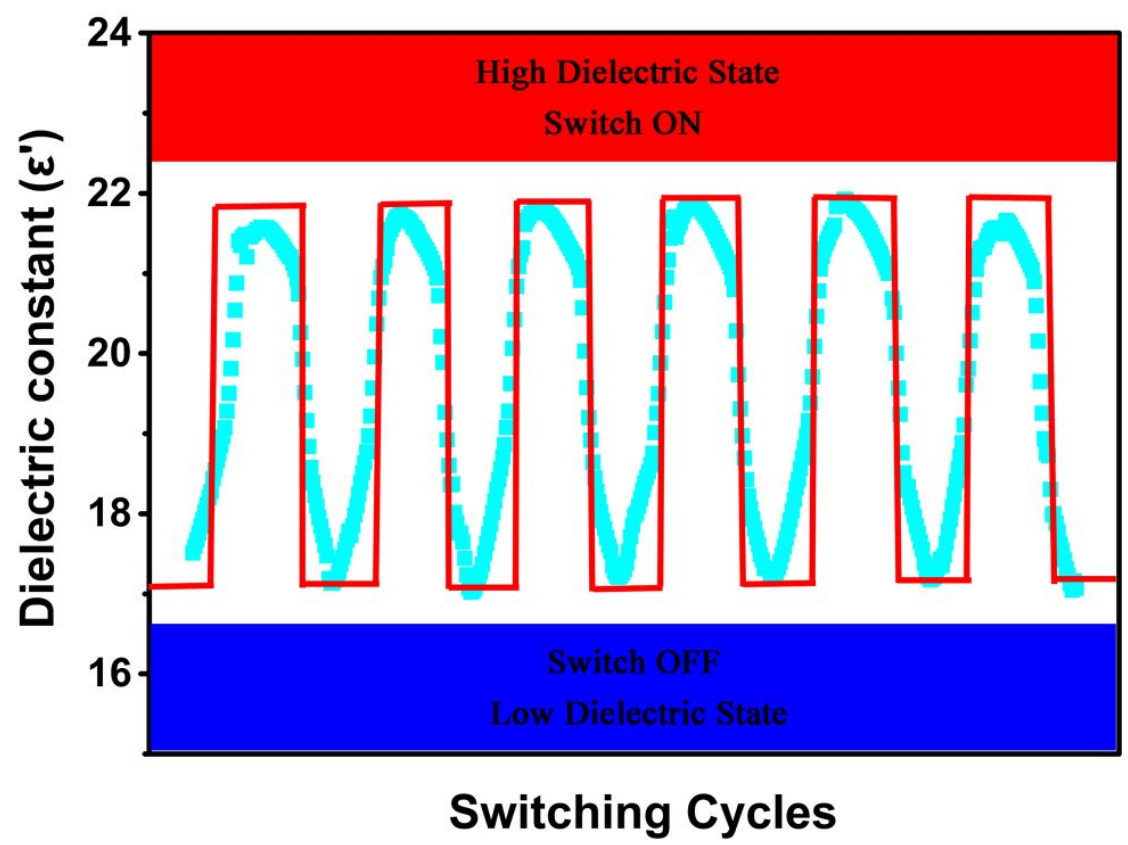

Figure S8. Reversible switching effect of dielectric constant on single crystals of 1 along the $\mathrm{c}$ axis. The blue line represents the actual measured dielectric constant over time and the red line represents their fitting curves. 


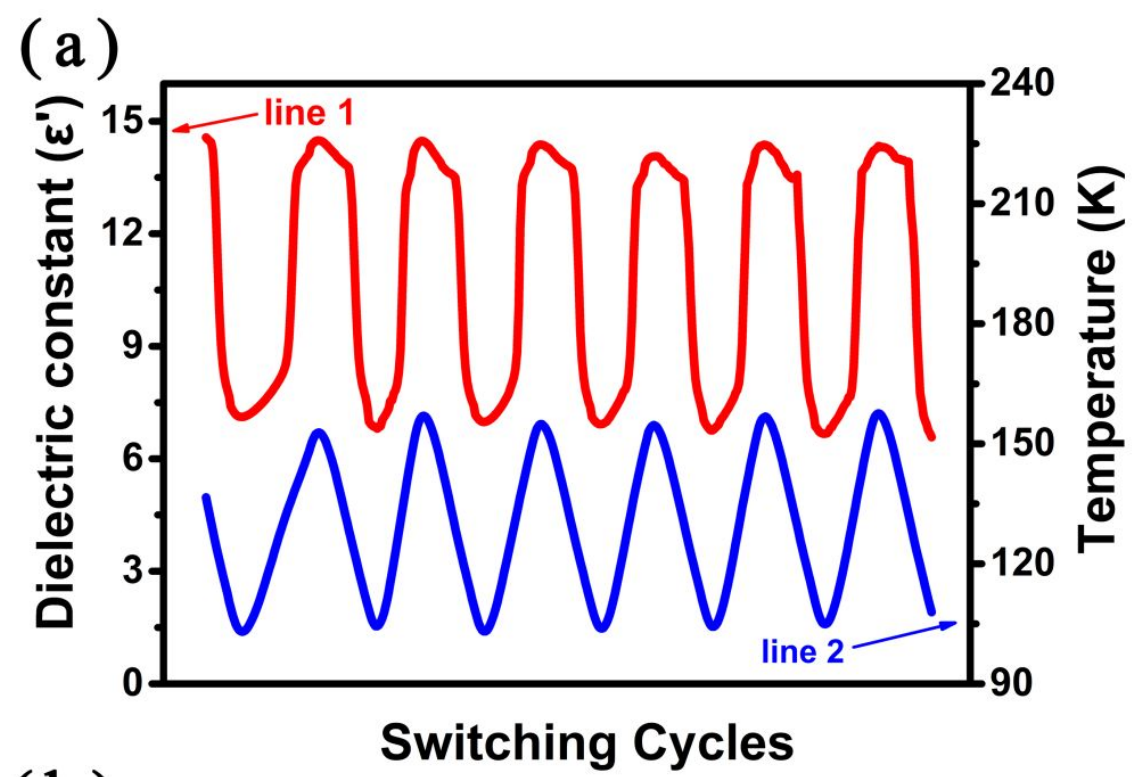

(b)

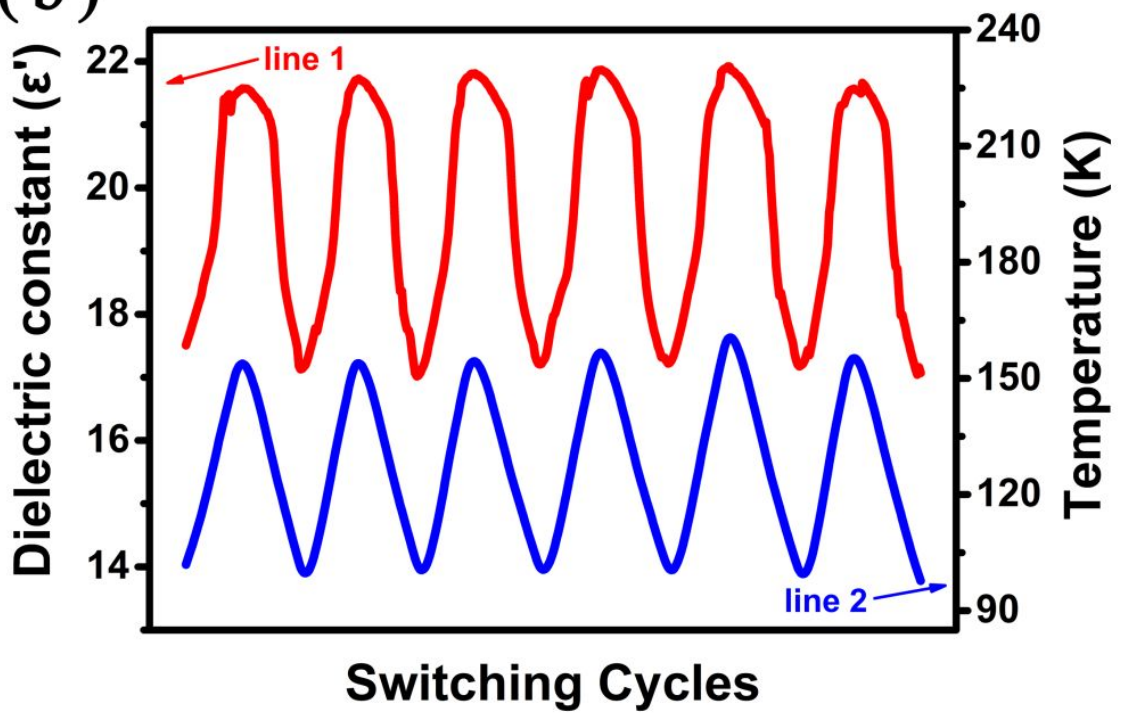

Figure S9. (a) Reversible switching effect of dielectric constant on polycrystalline of 1 (red line). And the corresponding temperature curve is red line 2, indicating the relationship of dielectric switching curves and temperature profiles over time is matched the switches. (b) Reversible switching effect of dielectric constant on single crystals of $\mathbf{1}$ along the c axis. And the corresponding temperature curve is blue line 2, indicating the relationship of dielectric switching curves and temperature profiles over time is matched the switches. 
Table S1. Crystal data and structure refinements for 1 at $293 \mathrm{~K}$ and $418 \mathrm{~K}$.

\begin{tabular}{|c|c|c|}
\hline & LTP (293K) & HTP (418K) \\
\hline Empirical formula & $\mathrm{C}_{7} \mathrm{H}_{16} \mathrm{CdCl}_{3} \mathrm{~N}$ & $\mathrm{C}_{7} \mathrm{H}_{16} \mathrm{CdCl}_{3} \mathrm{~N}$ \\
\hline Formula weight & 332.96 & 332.96 \\
\hline Temperature $(\mathrm{K})$ & $293(2)$ & $418(2)$ \\
\hline Crystal system & Monoclinic & Hexagonal \\
\hline Space group & $P 2(1) / c$ & $P 6(3) / m m c$ \\
\hline$a(\AA)$ & $7.3001(3)$ & $9.9670(11)$ \\
\hline $\mathrm{b}(\AA)$ & $9.4434(4)$ & $9.9670(11)$ \\
\hline $\mathrm{c}(\AA)$ & $17.8004(6)$ & $6.8583(7)$ \\
\hline$\alpha\left(^{\circ}\right)$ & 90.00 & 90.00 \\
\hline$\beta\left(^{\circ}\right)$ & $91.041(3)$ & 90.00 \\
\hline$\gamma\left({ }^{\circ}\right)$ & 90.00 & 120.00 \\
\hline $\operatorname{Volume}\left(\AA^{3}\right)$ & $1226.92(8)$ & $590.03(11)$ \\
\hline $\mathrm{Z}$ & 4 & 2 \\
\hline Radiation type & Mo-K $\alpha$ & Mo-Ka \\
\hline Absorption correction & Multi-scan & Multi-scan \\
\hline $\mathrm{D}_{\text {calc }} / \mathrm{g} \mathrm{cm}^{-3}$ & 1.803 & 1.783 \\
\hline$F(000)$ & 656 & 296 \\
\hline GOF & 1.065 & 0.953 \\
\hline$R_{1}[I>2 \sigma(I)]$ & 0.0448 & 0.0634 \\
\hline $\mathrm{w} R 2[I>2 \sigma(I)]$ & 0.1229 & 0.2508 \\
\hline
\end{tabular}

Table S2. Selected bond lengths $[\AA]$ and angles $\left[{ }^{\circ}\right]$ for $\mathbf{1}$ at $293 \mathrm{~K}$ and $418 \mathrm{~K}$.

\begin{tabular}{|c|c|c|c|c|}
\hline Temperature & \multicolumn{2}{|c|}{ bond lengths $[\AA]$} & \multicolumn{2}{|c|}{ bond angles $\left[{ }^{\circ}\right]$} \\
\hline \multirow[t]{8}{*}{$293 K$} & $\mathrm{Cd} 1-\mathrm{Cl1}$ & $2.7479(10)$ & $\mathrm{C} 11^{\mathrm{i}}-\mathrm{Cd} 1-\mathrm{Cl1}$ & $83.99(3)$ \\
\hline & $\mathrm{Cd} 1-\mathrm{Cl1}^{\mathrm{i}}$ & $2.4722(9)$ & $\mathrm{Cl} 11^{\mathrm{i}}-\mathrm{Cd} 1-\mathrm{Cl} 3$ & $93.70(3)$ \\
\hline & $\mathrm{Cd} 1-\mathrm{Cl} 2$ & $2.4506(10)$ & $\mathrm{Cl} 11^{\mathrm{i}}-\mathrm{Cd} 1-\mathrm{Cl}^{3 \mathrm{ii}}$ & $125.95(4)$ \\
\hline & $\mathrm{Cd} 1-\mathrm{Cl} 3$ & $2.7055(10)$ & $\mathrm{Cl} 2-\mathrm{Cd} 1-\mathrm{Cl} 1$ & $91.74(4)$ \\
\hline & $\mathrm{Cd} 1-\mathrm{Cl} 3^{\mathrm{ii}}$ & $2.4761(9)$ & $\mathrm{Cl} 2-\mathrm{Cd} 1-\mathrm{C} 11^{\mathrm{i}}$ & $117.87(4)$ \\
\hline & $\mathrm{Cl} 1-\mathrm{Cd} 1^{\mathrm{i}}$ & $2.4722(9)$ & $\mathrm{Cl} 2-\mathrm{Cd} 1-\mathrm{Cl} 3$ & $94.40(4)$ \\
\hline & $\mathrm{Cl} 3-\mathrm{Cd} 1^{\mathrm{ii}}$ & $2.4761(9)$ & $\mathrm{Cl} 2-\mathrm{Cd} 1-\mathrm{Cl} 3^{\mathrm{ii}}$ & $116.14(5)$ \\
\hline & $\mathrm{C} 1-\mathrm{C} 2$ & $1.494(6)$ & $\mathrm{Cl} 3-\mathrm{Cd} 1-\mathrm{Cl} 1$ & $173.82(3)$ \\
\hline
\end{tabular}




\begin{tabular}{|c|c|c|c|c|}
\hline & $\mathrm{C} 3-\mathrm{C} 2$ & $1.531(7)$ & $\mathrm{Cl} 3^{\mathrm{ii}}-\mathrm{Cd} 1-\mathrm{Cl} 1$ & $92.45(3)$ \\
\hline & $\mathrm{C} 3-\mathrm{C} 4$ & $1.513(7)$ & $\mathrm{Cl} 3^{\mathrm{ii}}-\mathrm{Cd} 1-\mathrm{Cl} 3$ & $84.26(3)$ \\
\hline & $\mathrm{C} 4-\mathrm{C} 5$ & $1.501(7)$ & $\mathrm{Cd} 1-\mathrm{C} 11-\mathrm{Cd} 1$ & $96.01(3)$ \\
\hline & $\mathrm{C} 1-\mathrm{N} 1$ & $1.512(5)$ & $\mathrm{Cd} 11^{\mathrm{ii}}-\mathrm{Cl} 3-\mathrm{Cd} 1$ & $95.74(3)$ \\
\hline & $\mathrm{C} 5-\mathrm{N} 1$ & $1.518(5)$ & $\mathrm{C} 1-\mathrm{C} 2-\mathrm{C} 3$ & $111.4(4)$ \\
\hline & $\mathrm{C} 6-\mathrm{N} 1$ & $1.490(5)$ & $\mathrm{C} 4-\mathrm{C} 3-\mathrm{C} 2$ & $109.8(4)$ \\
\hline & $\mathrm{C} 7-\mathrm{N} 1$ & $1.478(5)$ & $\mathrm{C} 5-\mathrm{C} 4-\mathrm{C} 3$ & $111.7(4)$ \\
\hline & & & $\mathrm{C} 2-\mathrm{C} 1-\mathrm{N} 1$ & $112.9(4)$ \\
\hline & & & $\mathrm{C} 4-\mathrm{C} 5-\mathrm{N} 1$ & $113.1(4)$ \\
\hline & & & $\mathrm{C} 1-\mathrm{N} 1-\mathrm{C} 5$ & $108.5(3)$ \\
\hline & & & $\mathrm{C} 6-\mathrm{N} 1-\mathrm{C} 1$ & $111.9(3)$ \\
\hline & & & $\mathrm{C} 7-\mathrm{N} 1-\mathrm{C} 1$ & $108.8(3)$ \\
\hline & & & $\mathrm{C} 6-\mathrm{N} 1-\mathrm{C} 5$ & $110.6(3)$ \\
\hline & & & $\mathrm{C} 7-\mathrm{N} 1-\mathrm{C} 5$ & $109.2(3)$ \\
\hline & & & $\mathrm{C} 7-\mathrm{N} 1-\mathrm{C} 6$ & $107.8(4)$ \\
\hline \multirow[t]{6}{*}{$418 \mathrm{~K}$} & $\mathrm{Cd} 1-\mathrm{Cl1}$ & $2.66(2)$ & $\mathrm{Cl} 1^{\mathrm{v}}-\mathrm{Cd} 1-\mathrm{Cl}^{\mathrm{vi}}$ & $82.9(6)$ \\
\hline & $\mathrm{Cd} 1-\mathrm{Cl} 1^{\mathrm{iv}}$ & $2.66(2)$ & $\mathrm{Cl1}{ }^{\mathrm{iv}}-\mathrm{Cd} 1-\mathrm{Cl} 1$ & $82.9(6)$ \\
\hline & $\mathrm{Cd} 1-\mathrm{Cl}^{\mathrm{v}}$ & $2.66(2)$ & $\mathrm{C} 11^{\mathrm{v}}-\mathrm{Cd} 1-\mathrm{C} 11^{\mathrm{vii}}$ & $82.9(6)$ \\
\hline & $\mathrm{Cd} 1-\mathrm{Cl} 1^{\mathrm{vi}}$ & $2.66(2)$ & $\mathrm{Cl} 11^{\mathrm{vi}}-\mathrm{Cd} 1-\mathrm{Cl} 1^{\mathrm{vii}}$ & $82.9(6)$ \\
\hline & $\mathrm{Cd} 1-\mathrm{Cl} 1^{\mathrm{vii}}$ & $2.66(2)$ & $\mathrm{Cl} 1-\mathrm{Cd} 1-\mathrm{C} 11^{\mathrm{viii}}$ & $82.9(6)$ \\
\hline & $\mathrm{Cd} 1-\mathrm{Cl} 11^{\mathrm{viii}}$ & $2.66(2)$ & $\mathrm{Cl1}^{\mathrm{iv}}-\mathrm{Cd} 1-\mathrm{Cl1}$ vi & $97.1(6)$ \\
\hline
\end{tabular}




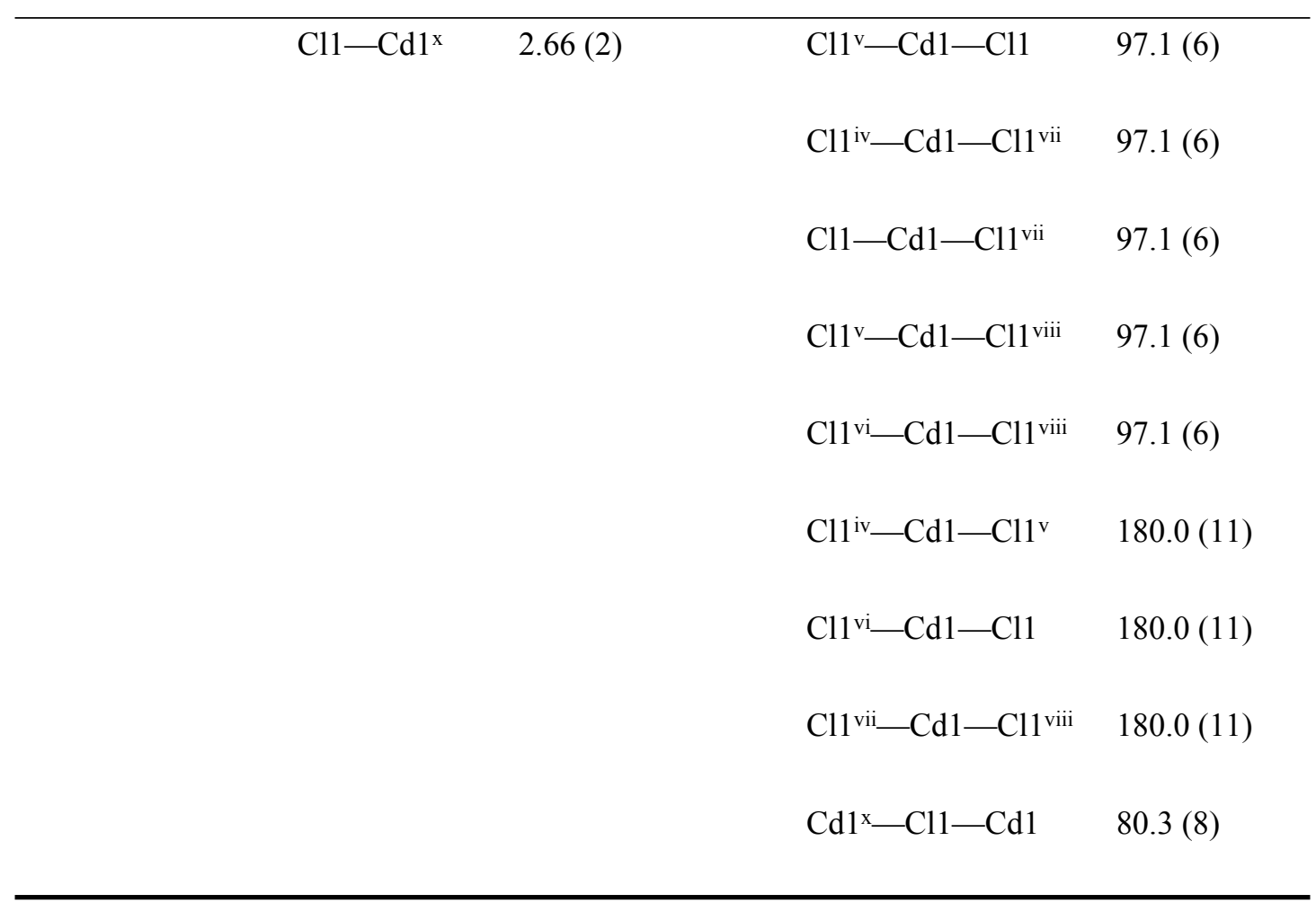

\section{Symmetry codes:}

$293 \mathrm{~K}$ (i) $-x,-y+1,-z$; (ii) $-x+1,-y+1,-z$.

$418 \mathrm{~K}$ (i) $-y+1, x-y, z$; (ii) $-x+y+1,-x+1, z$; (iii) $x, y,-z+3 / 2$; (iv) $-x+y+2,-x+1, z$; (v) $x-y, x-1,-z$; (vi) $-x+2,-y,-z$; (vii) $y+1,-x+y+1,-z$; (viii) $-y+1, x-y-1, z$; (ix) $-x+2,-y, z-1 / 2 ;(\mathrm{x})-x+2,-y, z+1 / 2$. 